\title{
Enhancement of Binding Affinity of Anti-Hapten Polyclonal IgG Recognizing Mitragynine using Affinity Purification
}

\author{
Radhiahtul Raehan Mustafa ${ }^{1}$, Rashidah Sukor ${ }^{1,2 *}$, Siti Mariam Mohd Nor ${ }^{3}$, \\ Nazamid Saari ${ }^{1}$, Farina Mustaffa Kamal ${ }^{4}$ and Aliah Zannierah Mohsin ${ }^{2}$ \\ ${ }^{1}$ Department of Food Science, Faculty of Food Science and Technology, Universiti Putra Malaysia, \\ 43400 UPM, Serdang, Selangor, Malaysia \\ ${ }^{2}$ Laboratory of Food Safety and Food Integrity, Institute of Tropical Agriculture and Food Security, \\ Universiti Putra Malaysia, 43400 UPM, Serdang, Selangor, Malaysia \\ ${ }^{3}$ Department of Chemistry, Faculty of Science, Universiti Putra Malaysia, 43400 UPM, \\ Serdang, Selangor, Malaysia \\ ${ }^{4}$ Department of Veterinary Pathology and Microbiology, Faculty of Veterinary Medicine, \\ Universiti Putra Malaysia, 43400 UPM, Serdang, Selangor, Malaysia
}

\begin{abstract}
Antibodies are glycoproteins found in peritoneal fluid, serum, and blood. The antibody-based assay has been used for broad applications such as immunodiagnostic and other biomedical applications. Depending on the intended application, a highly purified polyclonal antibody could be used as an alternative. Purification of antibodies from anti-sera has been proven as one of the methods to enhance the binding affinity of antibodies towards its antigen. We report herein the enhancement of the binding affinity of anti-hapten polyclonal IgG recognizing mitragynine using affinity purification. Serum from the terminal bleed of New Zealand White (NZW) rabbits immunized with mitragynine conjugated with cationizedbovine serum albumin at methyl ester (C22-MG-cBSA), or aromatic ether modification (C9-MG-cBSA) were subjected to HiTrap Protein G affinity purification using fast protein liquid chromatography (FPLC). The elution

ARTICLE INFO

Article history:

Received: 10 February 2021

Accepted: 05 July 2021

Published: 18 October 2021

DOI: https://doi.org/10.47836/pjst.29.4.11

E-mail addresses:

raehanmustafa@gmail.com (Radhiahtul Raehan Mustafa)

rashidah@upm.edu.my (Rashidah Sukor)

smariam@upm.edu.my (Siti Mariam Mohd Nor)

nazamid@upm.edu.my (Nazamid Saari)

farina@upm.edu.my (Farina Mustaffa Kamal)

aliahmohsin89@gmail.com (Aliah Zannierah Mohsin)

* Corresponding author

peak from chromatography fractions was analyzed using sodium dodecyl sulfatepolyacrylamide gel electrophoresis (SDSPAGE) and Western blot. Here, we report the binding of polyclonal antibodies produced from inoculation of either C22-MG-cBSA or C9-MG-cBSA immunogens of which mitragynine-ovalbumin (MG-OVA) was used as coating antigen in the ELISA assay. Non purified anti-sera from C22-MG-cBSA-
\end{abstract}


inoculated rabbits showed higher titer than C9-MG-cBSA at 1/128 000 and 1/32 000 dilutions, respectively. The affinity of purified poly-IgGs from rabbits immunized with C22-MG-cBSA showed a mean $\mathrm{K}_{d}$ value of $7.965 \times 10^{-6} \mu \mathrm{M}$, which was lower than those immunized with C9-MG-cBSA at mean $\mathrm{K}_{\mathrm{d}}$ of $1.390 \times 10^{-4} \mu \mathrm{M}$. In addition, the purified polyIgGs showed higher binding towards MG-OVA than non-purified anti-sera at comparable protein concentrations. These results indicated that the higher binding affinity of purified polyclonal IgG is due to the reduced competition among polyclonal antibodies with non$\mathrm{IgG}$ proteins that co-existed in the non-purified anti-sera after the affinity purification.

Keywords: Affinity chromatography, ELISA, IgG purification, mitragynine, polyclonal antibody

\section{INTRODUCTION}

Mitragynine is the main alkaloid in Mitragyna speciosa (kratom), which contributes to human addiction. It is utilized in the same context as other drugs and is often misused as a substitute for commercial drugs (Meireles et al., 2019). This psychoactive compound could render psychotropic and toxic effects. Therefore, the determination of mitragynine in human biological samples (e.g., saliva, urine, and blood) is important to monitor kratom misuse. Immuno-based analytical methods such as enzyme-linked immunosorbent assay (ELISA) and immunoblotting offer high advantages for straightforwardly detecting the alkaloid. However, a high sensitivity, specificity, reliability, and robustness of these analytical methods depend on the quality of the antibodies as biorecognition molecules. Having high affinity of antibodies could prevent the non-specific binding of other proteins in ELISA. Therefore, it is crucial for indirect ELISA format for hapten (mitragynine) that involves the coating of diluted antibodies onto the plate surface.

Antibodies or immunoglobulin $\mathrm{G}(\mathrm{IgG}) \mathrm{s}$ are generally isolated from serum, plasma, egg yolk, and peritoneal fluid, which generally co-exist with other biomolecules and proteins such as albumin and salt that may interfere with the binding between antibodies and the target antigen (Arora et al., 2016). This problem causes non-specific background in many analytical techniques such as ELISA, reducing the detection signal or producing a falsepositive result. Besides, antibodies with low sensitivity and specificity for their analyte can recognise other molecules structurally similar to the target analyte. Since kratom is usually consumed in a mixture of other substances, also known as kratom cocktails, the binding affinity of anti-mitragynine antibodies can be affected by kratom matrices or additives used in the cocktail. Thus, an efficient method for purifying antibodies is crucial for antibodies generation's high affinity and specificity.

Common techniques employed for antibody purification include ammonium sulphate precipitation, filtration, electrophoretic separation, and affinity chromatography (Tiller \& Tessier, 2015). The choice of techniques is based on their key attributes, including size, 
folding stability, solubility, binding affinity, isoelectric point (pI), and hydrophobicity of the antibody (Tiller \& Tessier, 2015). Affinity chromatography is an effective separation tool for purifying individual molecules from a complex mixture, removing any unwanted proteins while maintaining the desired product consistency. It is a promising method for purifying antibodies from various animals, which employs different types of binding molecules (ligands) and solid phase support (matrices). It has significant advantages, including ease of operation, rapid, high yield and purity $(>90 \%)$, and excellent selectivity towards the target molecule. This method has been reported to be the most efficient technique for purifying antibodies (Arora et al., 2016; Lopez et al., 2019, Fishman \& Berg, 2019).

Purification of antibodies from non-purified anti-sera has many advantages such as increased antibody concentration, purity, stability for long-term analysis and storage, as well as reduced non-specific background activities and variation lot to lot batch of the antibodies since polyclonal antibodies contain a mixture of the antibodies being produced (Fishman \& Berg, 2019; Tiller \& Tessier, 2015). However, the purification technique may and may not enhance the binding affinity of the antibody. As reported by Mustafaoglu et al. (2016), purification of antibodies did not improve the binding activity of the antibody due to the antibody activity (including both antigen detection and $\mathrm{Fc}$ recognition) was utterly preserved after purification. Limited studies were reported on the difference of binding affinity of purified poly-IgG or non-purified anti-sera in immunoassay. The choice of using purified antibodies or non-purified anti-sera in immuno-based assay depends on several factors, including the cost, availability of the target analyte in large quantity, personnel technical competency, and intended use of the assay. Therefore, this study aims to evaluate the binding affinity of non-purified anti-sera and purified poly-IgGs of anti-mitragynine polyclonal antibodies towards mitragynine conjugated with cationized-bovine serum albumin at different positions of mitragynine molecule, i.e., methyl ester (C22-MG-cBSA) and aromatic ether (C9-MG-cBSA).

\section{MATERIAL AND METHODS}

\section{Materials and Reagents}

All chemicals and reagents were of analytical grade. Mitragynine standard ( $98 \%$ purity) from Chromadex (Los Angeles, CA). Titermax Gold Adjuvant, Freund's Incomplete Adjuvant, ovalbumin (OVA), Tween 20 and 1-step ultra 3, 3', 5, 5'-tetramethylbenzidine (TMB) from Sigma Aldrich (St. Louis, MO). Alkaline phosphatase- and peroxidase-conjugated AffiniPure goat anti-rabbit $(\mathrm{H}+\mathrm{L})$ IgGs from Jackson ImmunoResearch Laboratories (West Grove, PA). Nitro blue tetrazolium chloride/5-bromo-4-chloro-3-indolyl phosphate (NBT/ BCIP) chromogen and immobilon-P PVDF membrane from Merck (Darmstadt, Germany). Enhanced chemiluminescent (ECL) and HiTrap Protein G HP column from GE Healthcare (Uppsala, Sweden). Nunc Maxisorp 96-well microtiter plates from Thermo Fisher Scientific 
(Waltham, MA). Coomassie Blue G-250, Tris, glycine and sodium dodecyl sulphate from Bio-Rad Laboratories (Hercules, CA). Ultra-pure water (18.2 M $\Omega . c m)$ from ELGA Lab Water (Lane End, UK).

\section{Immunization of Animal}

Animal studies were approved by the Institutional of Animal Care and Use Committee of Universiti Putra Malaysia (UPM/IACUC/AUP-R004). Mitragynine used as immunogen was modified at 16- $\mathrm{COOCH}_{3}$ and 9- $\mathrm{OCH}_{3}$ and conjugated to $\mathrm{cBSA}$ (Figure 1) according to Limsuwanchote et al. (2014) and Esteve-Turrillas et al. (2018) with slight modifications.

Four female 12-weeks old New Zealand White (NZW) rabbits (2 animals per immunogen) were immunized with the conjugated immunogen. The rabbit immunization protocol was carried out in accordance with polyclonal antibody production guidelines (Delahaut, 2017). Prior to the immunization procedure, rabbits were fed, primed and acclimatised for 14 days at the animal research facility, Faculty of Veterinary Medicine, Universiti Putra Malaysia, Serdang, Selangor. Pre-immune blood was collected prior to the first immunization and used as a negative control. For initial immunization, $500 \mu \mathrm{g} /$ $\mathrm{mL}$ of immunogen (MG-22-cBSA and MG-9-cBSA) was dissolved in $1 \mathrm{~mL} \mathrm{NaCl}(0.9 \%)$ and mixed with an equal volume of Titermax gold adjuvant. Rabbits were immunized subcutaneously (SQ) with $1 \mathrm{~mL}(0.25 \mathrm{~mL}$ per site for four sites) of the prepared solution. The immunogens were prepared at $250 \mu \mathrm{g} / \mathrm{mL}$ of antigen in Incomplete Freund's Adjuvant for subsequent immunization boosters. About five $\mathrm{mL}$ of blood per animal were collected after seven days of immunization via marginal ear vein. Rabbits were immunized at a one-month interval (28 days) with a total of 168 days of immunization protocol. Rabbits were euthanized by overdosage of pentobarbital via intracardiac injection. Approximately

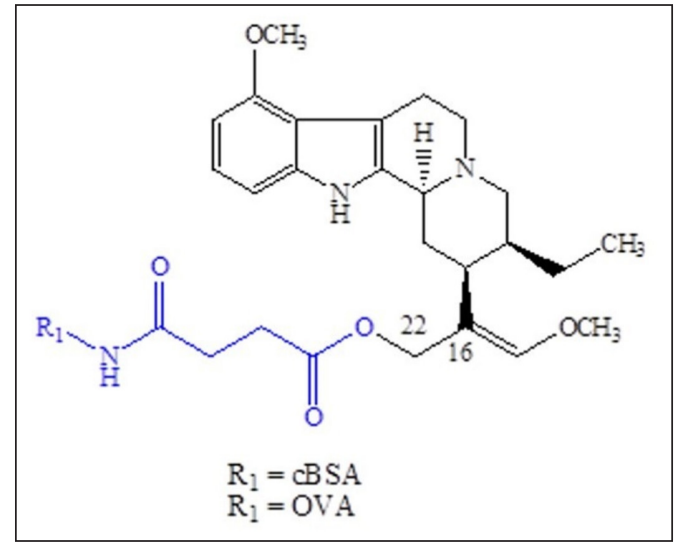

(a)

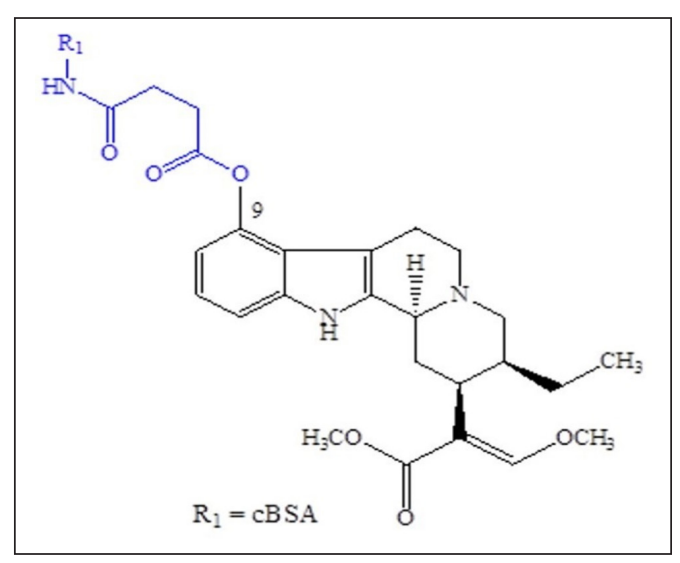

(b)

Figure 1. Mitragynine used as immunogen modified at two different molecule positions; (a) 16-COOCH3 and (b) 9-OCH3 and conjugated to cBSA. Mitragynine conjugated with OVA at 16-COOCH3 was used as coating antigen in ELISA 
80-120 mL of terminal sera per animal was collected through cardiac puncture. Blood was stored at $4^{\circ} \mathrm{C}$ (overnight) to clot and centrifuged for $20 \mathrm{mins}$ at $1000 \mathrm{rpm}$. The non-purified anti-sera were stored in aliquots at $-20^{\circ} \mathrm{C}$ until further use.

\section{Sodium Dodecyl Sulphate-Polyacrylamide Gel Electrophoresis (SDS-PAGE)}

The purity of purified poly-IgGs was examined after affinity chromatography by SDS-PAGE. According to the manufacturer's instructions, polyacrylamide (12\%) was used as separation gel in reducing conditions (Bio-Rad Laboratories, Hercules, CA). Purified poly-IgGs $(20 \mu \mathrm{g} / \mathrm{mL})$ were prepared in SDS-PAGE sample buffer containing 2-mercaptoethanol as a reducing agent and heated at $95^{\circ} \mathrm{C}$ for 10 mins. The sample was electrophoresed at $110 \mathrm{~V}$ for 60 mins and stained with $0.1 \%$ Coomassie Brilliant Blue G-250 for 30 mins, and subsequently destained with methanol: acetic acid (4:1, v/v) until the background was clear.

\section{Purification of Polyclonal IgGs}

Non-purified anti-sera of rabbits from terminal bleed $(10 \mathrm{~mL})$ were pooled and dialyzed against PBS ( $\mathrm{pH} 7.4)$ at $4^{\circ} \mathrm{C}$ with three times buffer changes. The non-purified anti-sera protein concentration was determined using NanoDrop ND-1000 (Thermo, Waltham, MA) at $280 \mathrm{~nm}$. The non-purified anti-sera were subjected to affinity chromatography on ÄKTA Purifier 10 of Fast Protein Liquid Chromatography system (GE Healthcare Bio-Sciences, Pittsburg, PA) for IgG purification according to the manufacturer's instruction. Briefly, the binding buffer (20 mM sodium phosphate, $\mathrm{pH} 7$ ) was mixed with an equal volume of the anti-mitragynine of non-purified anti-sera $(5 \mathrm{~mL})$, filtered $(0.45 \mu \mathrm{m})$ and loaded onto a $1 \mathrm{~mL}$ of HiTrap Protein G HP column through a sample loop. The column was preequilibrated with the binding buffer $(5 \mathrm{~mL})$. The specific $\mathrm{IgG}$ in the non-purified anti-sera solution was allowed to bind to the protein $G$ affinity column. After that, the unbound substances were washed by rinsing with the binding buffer at a flow rate of $1 \mathrm{~mL} / \mathrm{min}$ at a five-column volume $(5 \mathrm{~mL})$. Bound IgGs were eluted using glycine- $\mathrm{HCl}(0.1 \mathrm{M}, \mathrm{pH}$ 2.7) and neutralized with Tris- $\mathrm{HCl}$ (1 M, pH 9). Purified poly-IgGs were collected during the elution peak generated and dialyzed (MWCO 12-14,000 Da) against PBS (pH 7.4) at $4^{\circ} \mathrm{C}$ within 24 hours and lyophilized. The weight of lyophilized IgGs was recorded and stored at $-20^{\circ} \mathrm{C}$ until further use. The concentration of purified poly-IgGs was measured by NanoDrop ND-1000 at an absorbance value of $280 \mathrm{~nm}$ calculated using 1.35 as extinction co-efficient ( $\varepsilon$ ) for rabbit IgG (Zhao et al., 2019).

\section{Western Blot}

According to the manufacturer's instructions, purified poly-IgGs from SDS-PAGE were further identified on Western blot (Bio-Rad Laboratories, Hercules, CA). Briefly, the 
SDS gel was equilibrated in transfer buffer (5.76 g of Tris base, $2.95 \mathrm{~g}$ of glycine and 200 $\mathrm{mL}$ of methanol in $1 \mathrm{~L}$ of water) for 15 mins prior to transfer onto immobilon-P PVDF membrane. Next, a trans-blot semi-dry transfer cell (Bio-Rad Laboratories, Hercules, CA) was used to transfer the gel onto the membranes at $24 \mathrm{~V}$ and room temperature for 30 mins. Subsequently, non-specific binding on the membrane was blocked with skimmed milk (5\%, w/v) in PBS and incubated with gentle shaking for 1 hour. Next, the membrane was washed three times with PBST and probed with alkaline phosphatase-conjugated AffiniPure goat anti-rabbit $\operatorname{IgG}(\mathrm{H}+\mathrm{L})(1: 1000, \mathrm{v} / \mathrm{v})$. The membrane was further incubated with gentle shaking at room temperature for 2 hours. After the washing step, the protein bands were developed by the addition of NBT/BCIP substrate. The PVDF membrane was then rinsed with water and air-dried.

\section{Indirect Enzyme-Linked Immunosorbent Assay (ELISA)}

The humoral response of rabbit non-purified anti-sera of anti-mitragynine polyclonal antibodies was examined through indirect ELISA according to Mohsin et al. (2020), with slight modifications. The binding affinity of purified poly-IgGs from all rabbits was performed using total protein concentration at $5,2.5$ and $1.25 \mu \mathrm{g} / \mathrm{mL}$ of pre-immune antisera, non-purified anti-sera and purified poly-IgG from a terminal bleed of the rabbits. Ninety-six-well microtiter plates were coated with MG-OVA at $0.25 \mu \mathrm{g} / \mathrm{mL}(100 \mu \mathrm{L})$ in PBS (pH 7.4) and incubated overnight (16 hours) at $4^{\circ} \mathrm{C}$. The plates were washed three times using PBS and blocked using skimmed milk $(5 \% \mathrm{w} / \mathrm{v}, 250 \mu \mathrm{L})$ for 2 hours at room temperature with constant shaking $(100 \mathrm{rpm})$. Pre-immune anti-sera, non-purified antisera and purified poly-IgG with dilution in PBS $(100 \mu \mathrm{L})$ was added with total protein concentration at $5,2.5$ and $1.25 \mu \mathrm{g} / \mathrm{mL}$, and incubated for 1 hour at $37^{\circ} \mathrm{C}$. Next, goat anti-rabbit-HRP was added at $1 / 2500(\mathrm{v} / \mathrm{v})(0.16 \mu \mathrm{g} / \mathrm{mL})$ and further incubated at similar condition. The plates were washed three times with PBST (PBS containing 0.01\% Tween $20,250 \mu \mathrm{L})$ between incubation. The ELISA reaction was initiated by adding 1-Step ${ }^{\mathrm{TM}}$ Ultra 3,3',5,5'-tetramethylbenzidine (TMB) substrate solution $(100 \mu \mathrm{L})$ and the reaction was developed at room temperature for 20-30 mins in the dark. The reaction was quenched by the addition of $0.1 \mathrm{~N} \mathrm{HCl}(100 \mu \mathrm{L})$. The absorbance was measured using a Multiskan FC microplate reader (Thermo Scientific, Waltham, MA) at $450 \mathrm{~nm}$.

\section{Data Analysis}

All the samples were performed in triplicates $(n=3)$. The absorbance of pre-immune antisera was used as a control $\left(\mathrm{A}_{\mathrm{o}}\right)$. Results were analyzed using GraphPad Prism 5 (San Diego, $\mathrm{CA})$ and one-site binding formula, $\left[\mathrm{Y}=\mathrm{Bmax} * \mathrm{X} /\left(\mathrm{K}_{\mathrm{d}}+\mathrm{X}\right)\right]$ to evaluate the affinity constant $\left(\mathrm{K}_{\mathrm{d}}\right)$ of purified poly-IgGs. 


\section{RESULTS AND DISCUSSION}

\section{Purification of IgGs}

Purification of IgGs from non-purified antisera by protein $\mathrm{G}$ can be observed based on the solvent conductivity $(\mathrm{mS} / \mathrm{cm})$ and absorbance (mAU). The chromatographic profile, which showed the optimal condition of $\mathrm{IgG}$ purification, was in agreement with previous studies (Mohsin et al., 2020; Kang et al., 2016; Haddad et al., 2016). Figure 2 shows the chromatographic profile of the purified poly-IgGs using protein $\mathrm{G}$ affinity chromatography.

Approximately $1.49,2.32,2.16$, and $1.73 \mathrm{mg} / \mathrm{mL}$ of purified poly-IgGs were obtained from $10 \mathrm{~mL}$ of non-purified antisera from the terminal bleed of rabbit 1,2 , 3 , and 4, respectively. During purification, $\mathrm{pH}$ plays an important role to determine the purity and attributes of the purified-poly IgGs. The use of extreme $\mathrm{pH}$ (i.e., acidic or basic condition) in purification may degrade the IgGs, thus compromising the antigen recognition. This condition may also affect the functionality of the purified poly-IgGs (Lopez et al., 2019). A neutral $\mathrm{pH}$ of the binding buffer ( $\mathrm{pH} 7.0,20 \mathrm{mM}$ sodium phosphate) was used due to the favourable condition of the binding at a $\mathrm{pH}$ close to physiological condition between protein-G and the antibody. Neutral condition of binding buffer (potassium phosphate) is essential for optimal antibody-antigen binding. Protein $\mathrm{G}$ was allowed to bind to IgGs in the non-purified anti-sera for 10 min (peak 1) with the binding buffer. During this step, IgG adsorption to protein $\mathrm{G}$ occurred through affinity attachment at a low flow rate of 1 $\mathrm{mL} / \mathrm{min}$. A longer incubation period using a low flow rate during the binding process is necessary for the maximum binding of the IgG with the protein G (Arora et al., 2017).

After binding IgG to protein $\mathrm{G}$, the unbound non-specific molecule or weakly bound IgGs were further eliminated by washing the protein $\mathrm{G}$ column with 5 to $10 \mathrm{CV}$ binding buffer. The unbound materials were eliminated, and the bound IgG was recovered by changing the buffer to acidic conditions. At this step, impurities and non-specific binding from other molecules in biological samples were successfully removed (Lopez et al., 2019). The elution step of affinity chromatography can be performed using a competitive ligand or non-specifically, such as adjusting the polarity of the solution, $\mathrm{pH}$, ionic strength, and biomolecular chemical characteristics. The elution (peak 2) was performed by changing the ionic strength of the solution with the use of an elution buffer of $0.1 \mathrm{M}$ glycine-HCl. 
Increased salt concentration in the elution step elevated the ionic strength and weakened the protein G-IgG interactions (Mohsin et al., 2020).

Protein G-IgG binding dissociated by the positive charge of $\mathrm{HCl}$ at the acidic condition of the elution buffer. Therefore, during the elution step, the $\mathrm{pH}$ of the buffer was changed into acidic conditions using an elution buffer of $\mathrm{pH}$ 2.7. Acidic elution is most commonly used to elute purified poly-IgG due to favourable desorption and applies to most immunoglobulin species (Lopez et al., 2019). Nevertheless, the harsh acidic condition may lead to denaturation, alter the antigen-binding and conformational change, which cause aggregation to the IgGs (Arora et al., 2016). It was addressed by adding $1 \mathrm{M}$ Tris-HCl ( $\mathrm{pH}$ 9), which neutralized the IgGs condition, prevented loss of the IgGs activity, and maintained the IgGs structure.

\section{SDS-PAGE and Western Blot of Purified Poly-IgGs}

The purified poly-IgGs was assessed using SDS-PAGE and Western blotting under reducing condition. Figure 3 shows purified poly-IgG from four rabbits with two distinctive bands of the heavy and light chain of $\mathrm{IgG}$ at molecular weights of 50 and $25 \mathrm{kDa}$, respectively.

The basic structure of an antibody is made up of two identical heavy $(\mathrm{H})$ chains $(50$ $\mathrm{kDa})$ and light $(\mathrm{L})$ chains $(25 \mathrm{kDa})$ linked by disulfide bonds. The molecular weight of $\mathrm{IgG}$ in a non-reducing condition of SDS-PAGE is at $150 \mathrm{kDa}$ (Arora et al., 2016). Using 2-mercaptoethanol in the sample buffer as a reducing agent caused the dissociation of disulphide bonds on the antibody structure. In addition, the evaluation of purified poly-IgGs allows for the observation of contamination with other serum proteins. In Figure 3, there was an absence of additional bands in the SDS-PAGE and immunoblot, which verified the purity of the IgG from the rabbit of non-purified anti-sera. These results were according to

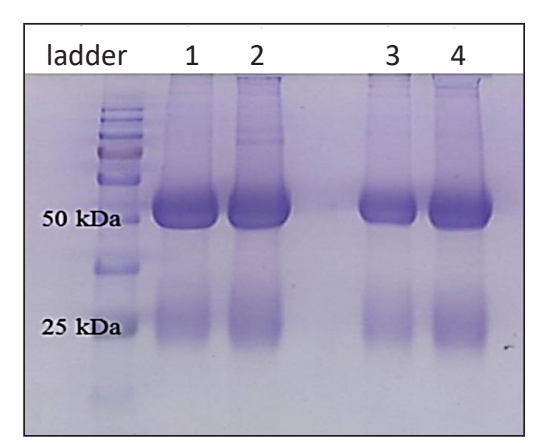

(a)

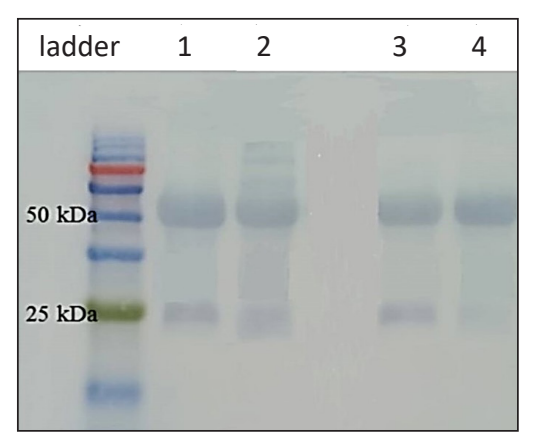

(b)

Figure 3. Characterisation of purified anti-mitragynine poly-IgGs. Two bands of 50 and $25 \mathrm{kDa}$, corresponding to the IgG heavy and light chains were shown on (a) SDS-PAGE (12\% separating gel), and (b) Western blot developed on NBT-BCIP substrate. Lane 1 and 2: purified poly-IgGs of rabbit 1 and 2 immunised with C22-MG-cBSA; Lane 3 and 4: purified poly-IgGs of rabbit 3 and 4 immunised with C9-MG-cBSA. Each well was loaded with $5 \mu \mathrm{g} / \mathrm{mL}$ of purified poly-IgG 
the previous studies, in which the pure IgG was obtained from polyclonal antibodies through affinity chromatography (Kang et al., 2016; Sadeghi et al., 2018, Mustafaoglu et al., 2016).

\section{Binding Comparison of Non-Purified Anti-Sera and Purified Poly-IgGs towards Mitragynine}

Purification of IgGs from anti-sera through affinity chromatography improved the binding affinity of the IgGs towards mitragynine at both C22- and C9-conjugated mitragynine (Figure 4). In addition, the purification was efficient in removing non-specifically bound protein to the ELISA plate, which interrupted the IgG-mitragynine binding. The binding of purified poly-IgGs from the four rabbits was compared with non-purified anti-sera from the terminal bleed by indirect ELISA, as shown in Figure 4.

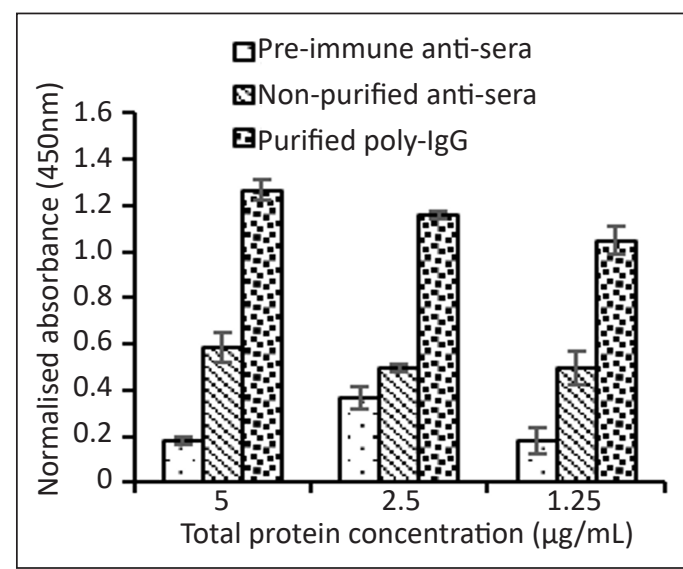

(a)

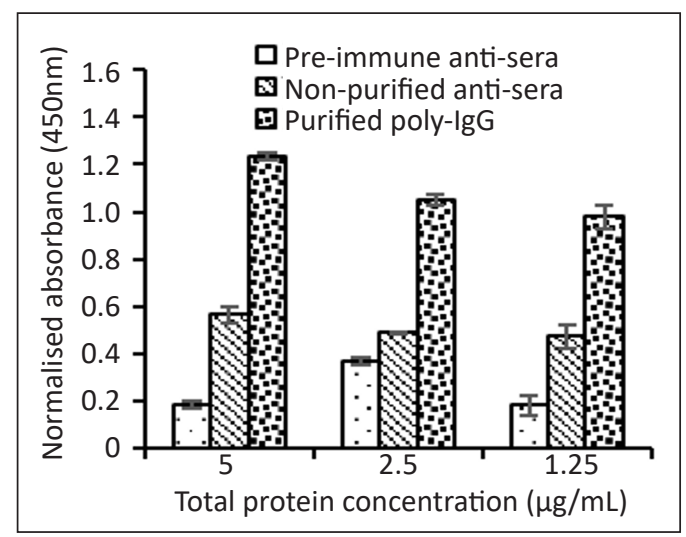

(c)

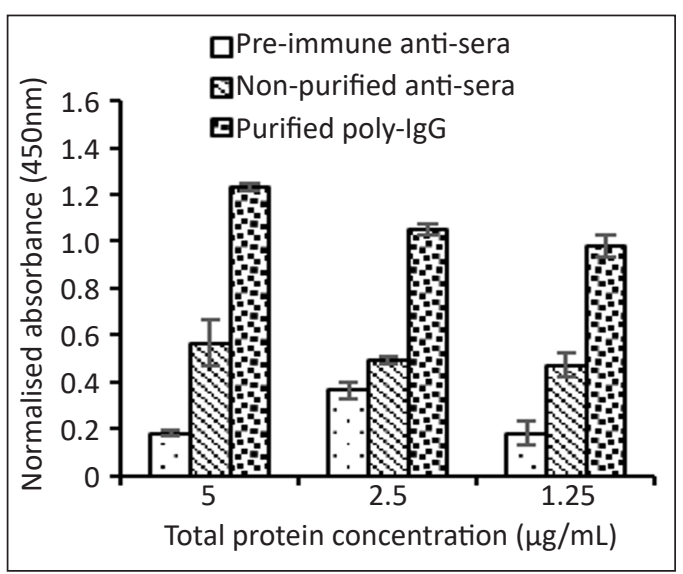

(b)

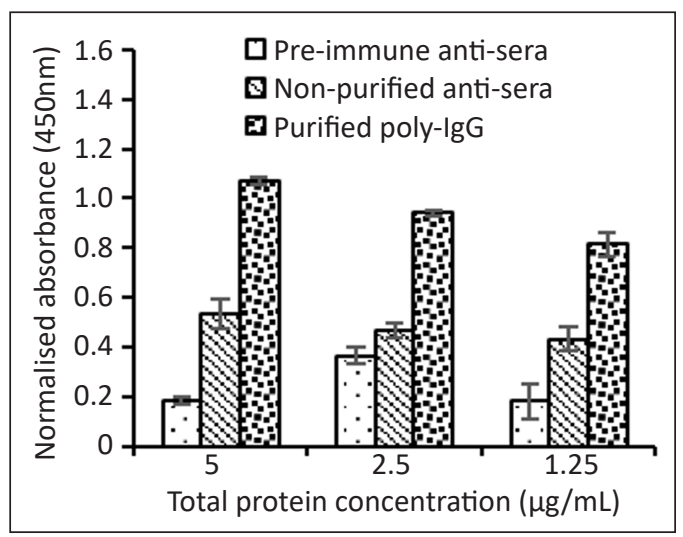

(d)

Figure 4. Binding comparison of non-purified anti-sera and purified poly-IgG of (a) rabbit 1 and (b) rabbit 2 immunized with C22-MG-cBSA and (c) rabbit 3 and (d) rabbit 4 immunized with C9-MG-cBSA. The non-purified anti-sera and purified poly-IgGs were from the terminal bleed of the rabbits. Pre-immune anti-sera were used as negative control. Normalized absorbance were obtained by subtracting background absorbance of pre-immune anti-sera. Values represent mean \pm SD of three readings. 
Purified poly-IgGs showed a higher binding towards mitragynine-OVA than nonpurified anti-sera at similar protein concentrations, which indicated that the affinity purification had improved the IgG binding. In addition, it was reported that approximately $75 \%$ of total IgGs were contained in a normal rabbit serum (Barker \& Reisen, 2019). Hence, purification of the IgGs has successfully removed the unspecific protein, including serum albumin and other protein that co-existed in the non-purified anti-sera.

In the humoral response of IgG, the binding of anti-mitragynine poly-IgG in nonpurified anti-sera towards mitragynine-cBSA conjugates are influenced by several important features. Firstly, the characteristic of the conjugates requires mitragynine-specific B cells and mitragynine-specific helper T cells. In addition, specific portions of hapten and carrier protein need to be associated to evoke an immune response physically. Secondly, the interaction occurs when the helper T cells bind to the B lymphocytes that only express major histocompatibility complex (MHC) class II molecules. Thus, the main factor inducing the humoral response of hapten-protein conjugates is an intrinsic determinant (hapten) recognized by B cells, and the other determinant is associated with MHC class II (carrier protein) recognized by T cell (Sanchez-Trincado et al., 2017).

An antibody molecule binds to the part of the antigen, which is called an antigenic epitope. The antibody recognizes the antigen-binding epitope at the antigen-binding site, which can be linear or conformational. Approximately $90 \%$ of B-cells epitopes are conformational, and only a small percentage of native antigens contain linear B-cell epitopes (Sanchez-Trincado et al., 2017). The antigen-binding sites were formed by folding the heavy and light chain variable domains, and the hypervariable domain will determine its complementary to the antigen.

\section{The Binding Affinity of Purified Poly-IgGs}

The affinity of the antibody, $\mathrm{K}_{\mathrm{d}}$ is referred to the strength of the antibody binding towards its antigen. The higher binding affinity of the antibody indicates the lower $\mathrm{K}_{\mathrm{d}}$ value (Laguna et al., 2015). Most small-sized antigens or haptens, such as toxins, hormones, and alkaloids, possess a single epitope that binds to each antibody. Therefore, the strength of the binding is determined solely by the affinity of the antibody towards the antigenic epitope. The $\mathrm{K}_{\mathrm{d}}$ value for polyclonal antibodies is an average of the total IgGs, is either weaker or stronger than the average. Figure 5 depicts the $\mathrm{K}_{\mathrm{d}}$ value of purified poly-IgGs from the four rabbits after affinity purification.

Based on Figure 5, mean $\mathrm{K}_{\mathrm{d}}$ of purified poly-IgGs antibody showed higher binding affinity from rabbits immunized with $\mathrm{C} 22-\mathrm{MG}-\mathrm{cBSA}$ at $7.965 \times 10^{-6} \mu \mathrm{M}$ and rabbits immunized with C9-MG-cBSA cBSA at of $1.390 \times 10^{-4} \mu \mathrm{M}$. It indicates that purification of poly-IgG from the non-purified anti-sera significantly enhanced the binding affinity of 


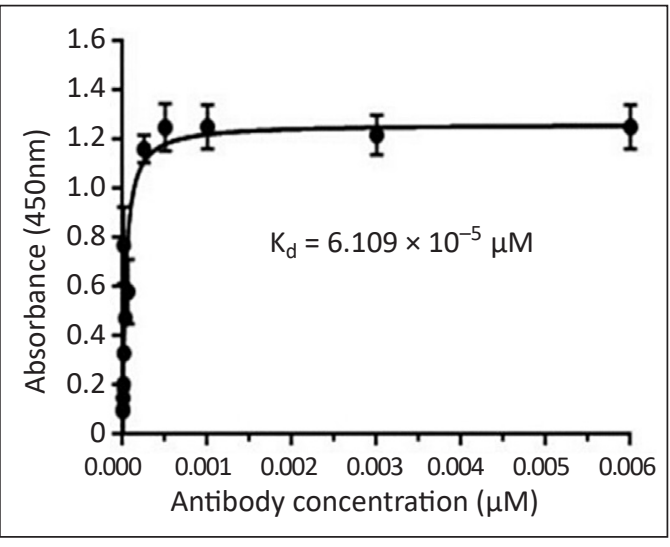

(a)

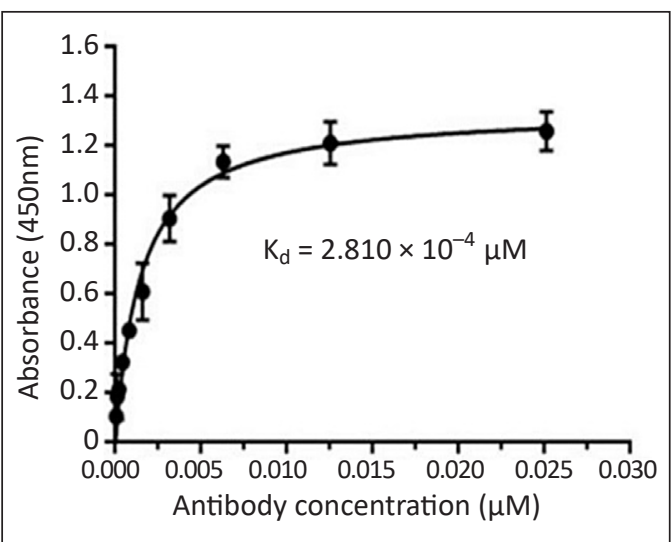

(c)

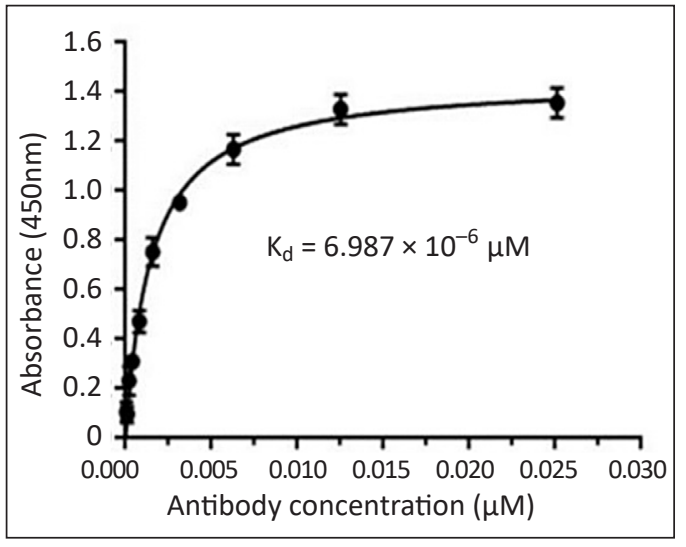

(b)

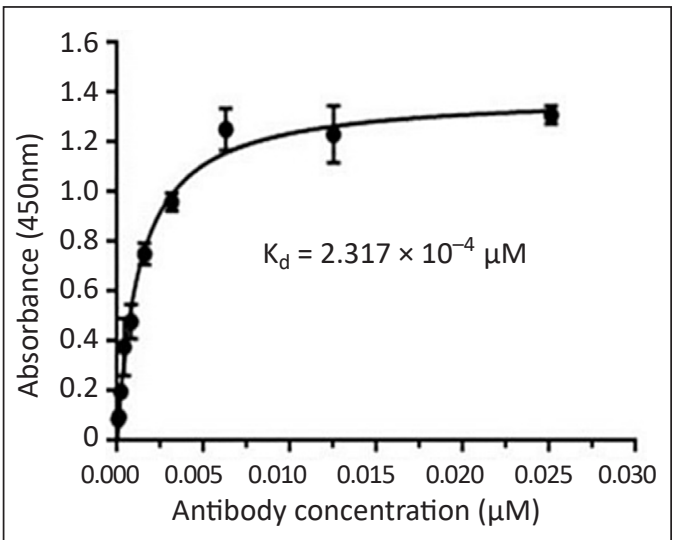

(d)

Figure 5. Binding affinity of purified poly-IgG for (a) rabbit 1 and (b) rabbit 2 immunized with C22-MGcBSA and (c) rabbit 3 and (d) rabbit 4 immunized with C9-MG-cBSA.

the IgG towards the target antigen, i.e. mitragynine. The result obtained was in line with a previous study that reported that affinity purification of $\mathrm{IgG}$ from non-purified anti-sera enhanced the binding affinity of antibodies towards peptides (Mohsin et al., 2020).

In affinity purification of $\mathrm{IgG}$, protein A and protein G, expressed in Staphylococcus aureus and Streptococcus sp., respectively, are the most widely employed immunoglobulinbinding proteins (Fishman \& Berg, 2019). It is due to the selectivity of the proteins towards $\mathrm{IgG}$ and the ability of the proteins to bind specifically to the $\mathrm{F}_{\mathrm{c}}$ region of $\mathrm{IgG}$ (Choe et al., 2016). However, protein A has only two binding sites which can react to the antibodies. Furthermore, the interaction of protein $A$ is not comparable to protein $G$ for all the animal species. As a result, protein G's ability to bind and characteristics make it suitable for monoclonal and polyclonal antibody purification with lower affinity for protein A (Walls \& Loughran, 2017). 
Additionally, protein $\mathrm{G}$ has a high IgG binding specificity of many mammalian species (Choe et al., 2016). Therefore, it is a better choice due to its ability to bind to a broader range of $\mathrm{IgG}$ from eukaryotic species and $\mathrm{IgG}$ classes. Besides, specific binding of protein $\mathrm{G}$ at the $\mathrm{F}_{\mathrm{c}}$ region of $\mathrm{IgG}$ in affinity chromatography exhibit minimal binding to albumin, resulting in high yield and high purified poly-IgG produced (Choe et al., 2016). The specificity binding of protein $\mathrm{G}$ to the $\mathrm{Fc}$ region of IgG makes them a good ligand for antibody purification.

Purification of IgG using affinity chromatography depends on the specific and reversible interaction between protein and its ligand, in this case, protein G and IgG. Protein G-affinity chromatography (coupled to agarose) using fast protein liquid chromatography (FPLC) is commonly used due to rapid and effective technique for the production of high purity antibodies (Fishman \& Berg, 2019). The purification process allows only specific target antigen from a complex mixture to bind to the protein-G. The HiTrap protein G column efficiently removes albumin and other proteins from non-purified anti-sera, resulting in a higher binding affinity of the antibody. The eluted antibodies can be obtained in high purity using different salt concentrations, $\mathrm{pH}$, and polarity (Arora et al., 2016).

\section{CONCLUSION}

Immunoglobulin $(\mathrm{IgG})$ is applied in ELISA in several forms such as total $\mathrm{IgG}$, serum, purified anti-sera, and specific IgG towards its antigen. Although polyclonal ELISA can be developed using non-purified anti-sera, this study had shown otherwise. The finding demonstrated that it is vital to purify antibodies to improve their affinity and prevent other protein that binds non-specifically to the low-signal plate of ELISA. A careful selection of IgG purification methods is also needed to avoid choosing non-representative populations of recovered IgGs. Since mitragynine $\mathrm{IgG}$ is not commercially available, the final product of purified anti-mitragynine $\mathrm{IgG}$ is an ideal and high-value biorecognition molecule in developing immuno-based detection methods such as ELISA. High-affinity anti-mitragynine IgG can also be a value-added molecule applied in portable sensor devices, which is yet to be reported. It will greatly benefit regulatory and enforcement agencies to monitor the misuse of kratom. Hence timely decisions can be made by the appropriate bodies.

\section{ACKNOWLEDGMENTS}

This work was financially supported by the Fundamental Research Grant Scheme (FRGS/2/2014/SG01/UPM/02/4) and MyBrain scholarship from the Ministry of Higher Education, Malaysia. 


\section{REFERENCES}

Arora, S., Saxena, V., \& Ayyar B. V. (2016). Affinity chromatography: A versatile technique for antibody purification. Methods, 116, 84-94. https://doi.org/10.1016/j.ymeth.2016.12.010

Barker, C. M., \& Reisen, W. K. (2019). Epidemiology of vector-borne diseases. In G. R. Mullen \& L. A. Durden (Eds.), Medical and veterinary entomology (pp. 33-49). Academic Press. https://doi.org/10.1016/ B978-0-12-814043-7.00004-2

Choe, W., Durgannavar, T. A., \& Chung, S. J. (2016). Fc-binding ligands of immunoglobulin G: An overview of high affinity proteins and peptides. Materials, 9(12), Article 994. https://doi.org/10.3390/ma9120994

Delahaut, P. (2017). Immunization - Choice of host, adjuvants and boosting schedules with emphasis on polyclonal antibody production. Methods, 116, 4-11. https://doi.org/10.1016/j.ymeth.2017.01.002

Esteve-Turrillas, F. A., Agulló, C., Mercader, J. V., Abad-Somovilla, A., \& Abad-Fuentes, A. (2018). Rationally designed haptens for highly sensitive monoclonal antibody-based immunoanalysis of fenhexamid. Analyst, 143(17), 4057-4066. https://doi.org/10.1039/C8AN00827B

Fishman, J. B., \& Berg, E. A. (2019). Antibody purification and storage. Cold Spring Harbor Laboratory Press. https://doi.org/10.1101/pdb.top099101Cold Spring Harb Protoc 2019

Haddad, M., Soukkarieh, C., Khalaf, H. E., \& Abbady, A. Q. (2016). Purification of polyclonal IgG specific for camelid's antibodies and their recombinant nanobodies. Open Life Sciences, 11, 1-9. https://doi. org/10.1515/biol-2016-0001

Kang, H. J., Choe, W., Min, J. K., Lee, Mi, Y., Kim, B. M., \& Chung, S. J. (2016). Cyclic peptide ligand with high binding capacity for affinity purification of immunoglobulin G. Journal of Chromatography A, 1466, 105-112. https://doi.org/10.1016/j.chroma.2016.09.007

Laguna, M., Holgado, M., Hernandez, A. L., Santamaría, B., Lavín, A., Soria, J., Suarez, T., Bardina, C., Jara, M., Sanza, F. J., \& Casquel, R. (2015). Antigen-antibody affinity for dry eye biomarkers by label free biosensing: Comparison with the ELISA technique. Sensors, 15(8), 19819-19829. https://doi.org/10.3390/ s150819819

Limsuwanchote, S., Wungsintaweekul, J., Keawpradub, N., Putalun, W., Morimoto, S., \& Tanaka, H. (2014). Development of indirect competitive ELISA for quantification of mitragynine in kratom (Mitragyna speciosa (Roxb.) Korth). Forensic Science International, 244, 70-77. https://doi.org/10.1016/j. forsciint.2014.08.011

Lopez, E., Scott, N. E., Wines, B. D., Hogarth, P. M., Wheatley, A. K., Kent, S. J., \& Chung, A. W. (2019). Low $\mathrm{pH}$ exposure during immunoglobulin $\mathrm{G}$ purification methods results in aggregates that avidly bind Fc $\gamma$ Receptors: Implications for measuring Fc dependent antibody functions. Frontiers in Immunology, 10, Article 2415. https://doi.org/10.3389/fimmu.2019.02415

Meireles, V., Rosado, T., Barroso, M., Soares, S., Gonçalves, J., Luís, Â, Caramelo D., Simao, A. Y., Fernandez, N., Duarte, A. P., \& Gallardo E. (2019). Mitragyna speciosa: Clinical, toxicological aspects and analysis in biological and non-biological samples. Medicine, 6(1), Article 35. https://doi.org/10.3390/ medicines 6010035 
Mohsin, A. Z., Sukor, R., Selamat, J., Hussin, A. S. M., Ismail, I. H., Jambari, N. N., \& Mustaffa-Kamal, F. (2020). Generation of high affinity anti-peptide polyclonal antibodies recognizing goat $\alpha_{\mathrm{s} 1}$-casein. Molecules, 25(11), Article 2622. https://doi.org/10.3390/molecules25112622

Mustafaoglu, N., Kiziltepe, T., \& Bilgicer, B. (2016). Antibody purification via affinity membrane chromatography method utilizing nucleotide binding site targeting with a small molecule. Analyst, 141(24), 6571-6582. https://doi.org/10.1039/C6AN02145J

Sadeghi, S., Aghebati-Maleki, L., Nozari, S., \& Majidi, J. (2018). A methodological approach for production and purification of polyclonal antibody against dog IgG. Veterinary Research Forum, 9(1), 13-18.

Sanchez-Trincado, J. L., Gomez-Perosanz, M., \& Reche, P. A. (2017). Fundamentals and methods for T- and B-cell epitope prediction. Journal Immunological Research, 8, 1-14. https://doi.org/10.1155/2017/2680160

Tiller, K. E., \& Tessier, P. M. (2015). Advances in antibody design. Annual Review of Biomedical Engineering, 17, 191-216. https://doi.org/10.1146/annurev-bioeng-071114-040733

Walls, D., \& Loughran, S. T. (Eds.). (2017). Protein chromatography: Methods and protocol. Humana Press.

Zhao, Y., Sun, X., Marquis, C. P., \& Lee, N. A. (2019). Development of a sensitive sandwich ELISA specific to $2 \mathrm{~S}$ albumin (Ana o 3 ) as a stable protein marker for cashew nut residue detection in pre-packaged food products. Food Control, 96, 432-440. https://doi.org/10.1016/j.foodcont.2018.09.038 